\title{
INTRAHEPATIC CHOLESTASIS OF PREgNANCY \\ AND ASSOCIATED MATERNAL-FETAL MORBIDITY \\ AND MORTALITY - EXPERIENCE OF SIX YEAR'S \\ OF A TERCIARY HOSPITAL
}

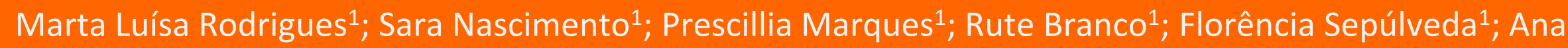

Cristina Costa ${ }^{1}$; Antónia Nazaré ${ }^{1}$.

1 - Obstetrics and Gynecology, Prof. Doctor Fernando Fonseca Hospital, Lisbon, Portugal.

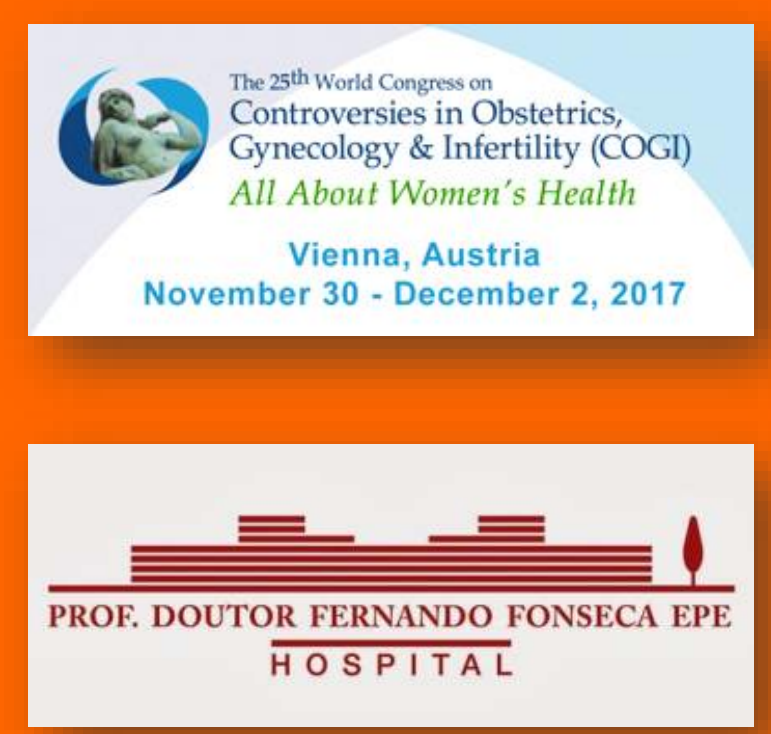

\section{PROBLEM STATEMENT}

The intrahepatic cholestasis of pregnancy (ICP) has an estimated incidence of $0,1-16 \%$ and is characterized by generalized pruritus (including hand palms and feet soles) associated with elevation total serum bile acids levels (BAL) and/or aminotransferases, in the second and third trimester of the pregnancy. Its etiology is unknown and the main associated complications are: preterm delivery, intrapartum fetal distress and, rarely, fetal death. The latter seems to be associated with BAL > 40 $\mu \mathrm{mol} / \mathrm{L}$. Ursodeoxycholic acid is used as a therapy for pruritus control, BAL normalization and prognosis improvement. Many authors suggest the delivery schedule 36-37 weeks of gestation. The recurrence rate in a subsequent pregnancy is $60-70 \%$.

\section{METHODS}

Retrospective descriptive study based on the analysis of the clinical processes of 47 pregnant women diagnosed with ICP in the last six years (2011-2016), at the Prof. Doctor Fernando Fonseca Hospital (Portugal, Lisbon). The analyzed variables were: antecedents of the pregnant woman, clinical condition associated with ICP, labour and data of the newborn.

Statistical analysis through Microsoft Excel @ 2010.

\section{RESULTS}

In this period, there were 47 cases of ICP whose average age was 28,8 years old ( $20-42$ years old).
Regarding the personal history of the pregnant woman, $20(42,6 \%)$ were multiparous, of whom 4 (20\%) had a history of ICP. (Graphic 1) At the time of ICP diagnosis, mean gestational age was 31,8 weeks (22-40 weeks) and BAL 40,6 $\mathrm{mol} / \mathrm{L}(10,8-180,1)$. Some 19 pregnant women $(40,4 \%) \mathrm{BAL}>40 \mu \mathrm{mol} / \mathrm{L}(40,4 \%)$, however, none were associated with fetal death. Concomitant to BA elevation, transaminases were elevated in 38 pregnant women $(80,6 \%)$. (Graphic 2)

Regarding delivery, the mean gestational age was 36,7 weeks (28-41 weeks). The cesarean delivery route occurred in 26 cases (53\%), 16 $(61,5 \%)$ of which in an emergency context due to a non-reassuring fetal state, suspected fetal-pelvic incompatibility or failure to induce labour. (Graphic 3-4)

Concerning complications, there were: 17 preterm delivery $(36,1 \%)$, whose average gestational age was 34,2 weeks (28-36); 10 cases of acute fetal distress $(21,3 \%)$ and no cases of fetal death. All newborns presented an Apgar Index $>7$ at the fifth minute.

\section{CONCLUSION}

The results obtained are mostly according to the most recent literature: diagnosis predominantly in the third trimester, elevation of aminotransferases concomitant to that of BAL, delivery after 36 weeks according to the gestational age at the time of diagnosis, preterm delivery and suffering fetal as more frequent complications and, finally, a non-negligible recurrence rate. However, no fetal death was observed even with high BAL values, which may be associated with an early intervention.
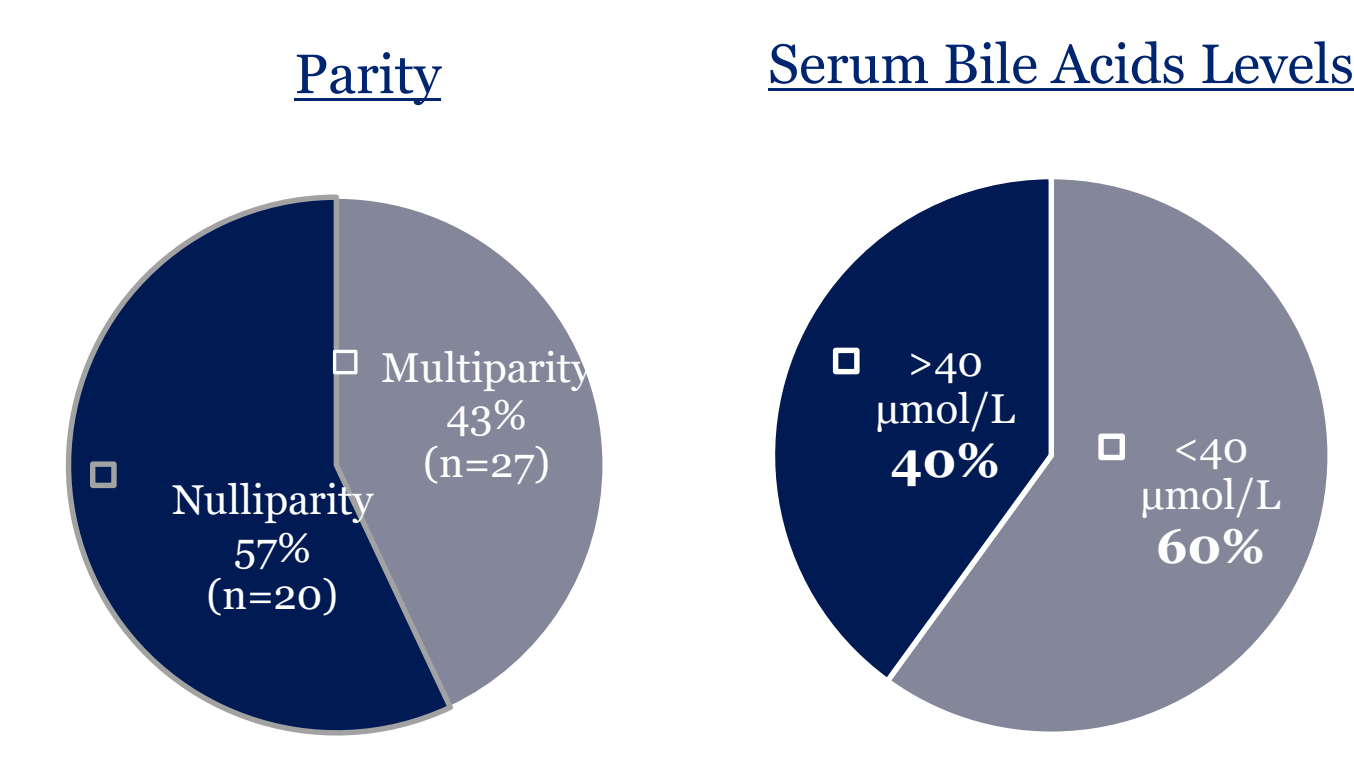

Graphic 1: Parity.
Graphic 2: Serum bile acids levels.

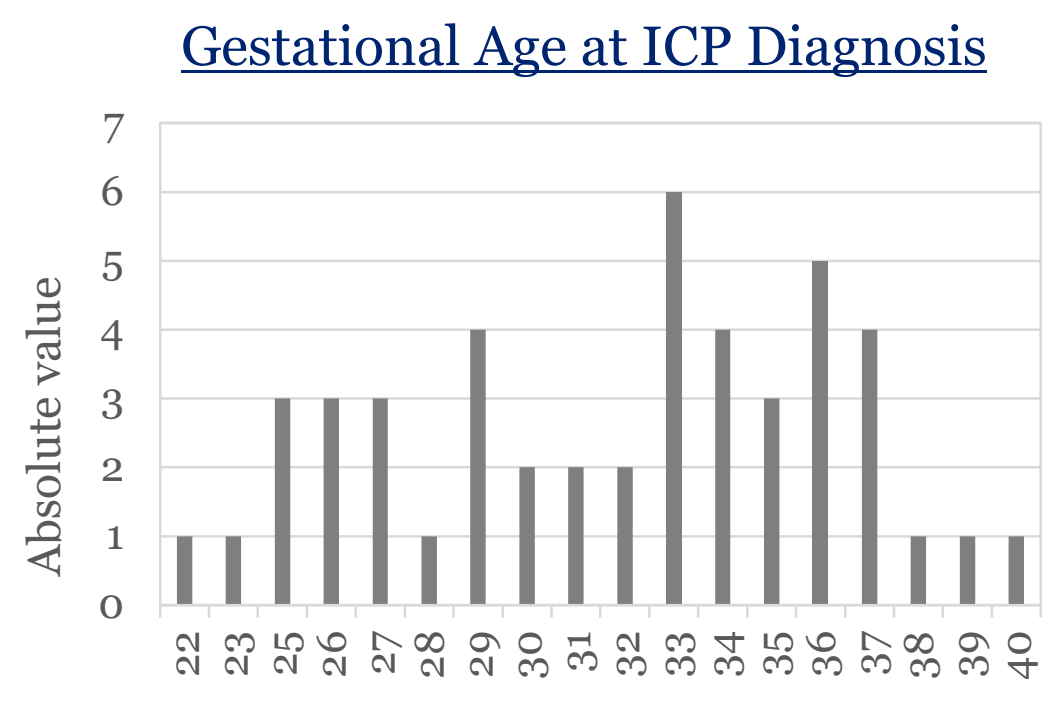

Gestational Age (weeks)

Graphic 3: Gestational age at ICP diagnosis.

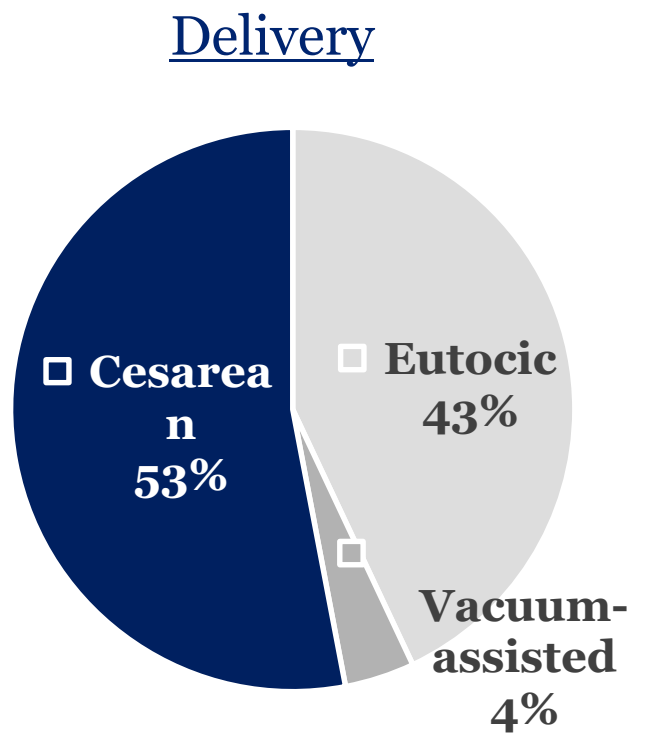

Graphic 4: Delivery. 